\title{
Chapter 10 \\ Development and Operation of a Carborne Survey System, KURAMA
}

\author{
Minoru Tanigaki
}

\begin{abstract}
A carborne survey system named as KURAMA (Kyoto University RAdiation MApping system) has been developed as a response to the nuclear accident at TEPCO Fukushima Daiichi Nuclear Power Plant. KURAMA is a $\gamma$-ray survey system with the global positioning system (GPS) and up-to-date network technologies developed for a primary use of carborne surveys. Based on the success of KURAMA, KURAMA-II, an improved version of KURAMA with better handling and ruggedness, is developed for the autonomous operation in public vehicles to minimize the workload of long-standing radiation monitoring required. Around two hundreds of KURAMA-II now serve for the continuous monitoring in residential areas by local buses as well as the periodical monitoring in Eastern Japan by the Japanese government. The outline and present status of KURAMA and KURAMA-II are introduced.
\end{abstract}

Keywords Radiometry $\bullet$ Mapping $\bullet \gamma$-ray $\bullet$ Carborne survey $\bullet$ Air dose rate - Fukushima Daiichi nuclear power plant

\subsection{Introduction}

The magnitude-9 earthquake in Eastern Japan on 11 March 2011 and the following massive tsunami caused the serious nuclear disaster of Fukushima Daiichi Nuclear Power Plant, which Japan had never experienced before. Huge amounts of radioactive isotopes were released in Fukushima and surrounding prefectures.

In such nuclear disasters, air dose rate maps are quite important to take measures to deal with the incident, such as assessing the radiological dose to the public, making plans for minimizing exposure to the public, and establishing procedures for environmental reclamation. The carborne $\gamma$-ray survey technique is known to be one of the effective methods to make air dose rate maps [2]. In this technique,

\footnotetext{
M. Tanigaki $(\bowtie)$

Research Reactor Institute, Kyoto University, 2-1010 Asashironishi, Kumatori, Osaka 590-0494, Japan

e-mail: tanigaki@rri.kyoto-u.ac.jp 
a continuous radiation measurement with location data throughout the subject area is performed by one or more monitoring cars equipped with radiation detectors. Unfortunately, the existing monitoring system didn't work well in the incident. Such monitoring cars tend to be multifunctional, thus too expensive to own multiple monitoring cars in a prefecture. Fukushima was the case, and to their worse, the only monitoring car and the data center were contaminated by radioactive materials released by the hydrogen explosions of the nuclear power plant. The monitoring cars owned by other prefectures were then collected, but such monitoring cars were too heavy to drive on heavily damaged roads in Fukushima. Then daily measurements of the air dose rate in the whole area of Fukushima were eventually performed by humans. The measuring personnel drove around more than 50 fixed points in Fukushima prefecture twice a day, and they measured the air dose rate of each point by portable survey meters. Airborne $\gamma$-ray surveys were performed by the Ministry of Education, Culture, Sports, Science and Technology of Japan (MEXT) and the US Department of Energy, but difficulties in the arrangement of aircraft, aviation regulations, and their flight schedules prevented immediate and frequent surveys in the areas of interest.

KURAMA was developed to overcome such difficulties in radiation surveys and to establish air dose-rate maps during the present incident. KURAMA was designed based on consumer products, enabling sufficient numbers of in-vehicle apparatus to be prepared within a short period. KURAMA realized high flexibility in the configuration of data processing hubs or in the arrangement of monitoring cars with the help of cloud technology. Based on the success of KURAMA, KURAMA-II was developed to realize the continuous monitoring in residential areas. An outline of KURAMA and KURAMA-II and their applications are presented.

\subsection{KURAMA}

KURAMA [10] is a $\gamma$-ray survey system with the global positioning system (GPS) and up-to-date network technologies developed for a primary use of carborne surveys. The system outline of KURAMA is shown in Fig. 10.1.

An in-vehicle unit of KURAMA consists of a conventional NaI scintillation survey meter with an appropriate energy compensation, an interface box for the analog voltage output from the detector to a USB port of PC, a GPS unit, a laptop PC, and a mobile Wi-Fi router (Fig. 10.2). Its simple and compact configuration allows users to set up an in-vehicle unit in a common automobile. The software of in-vehicle part is developed with LabVIEW. The radiation data collected every $3 \mathrm{~s}$ is tagged by its respective location data obtained by GPS and stored in a csv file. This csv files updated by respective monitoring cars are simultaneously shared with remote servers by Dropbox over a $3 \mathrm{G}$ network, unlike other typical carborne survey systems in which special telemetry systems or storage media are used for data 


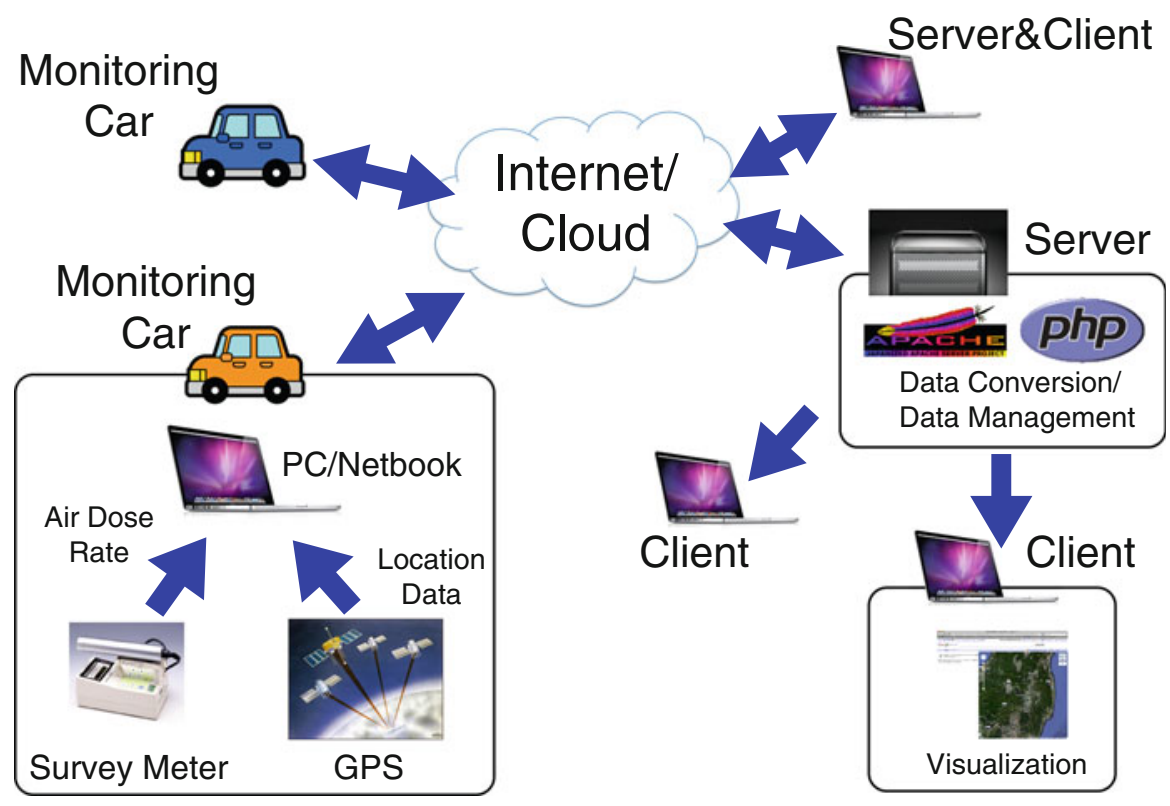

Fig. 10.1 The system outline of KURAMA. Monitoring cars and servers are connected over the Internet by cloud technology

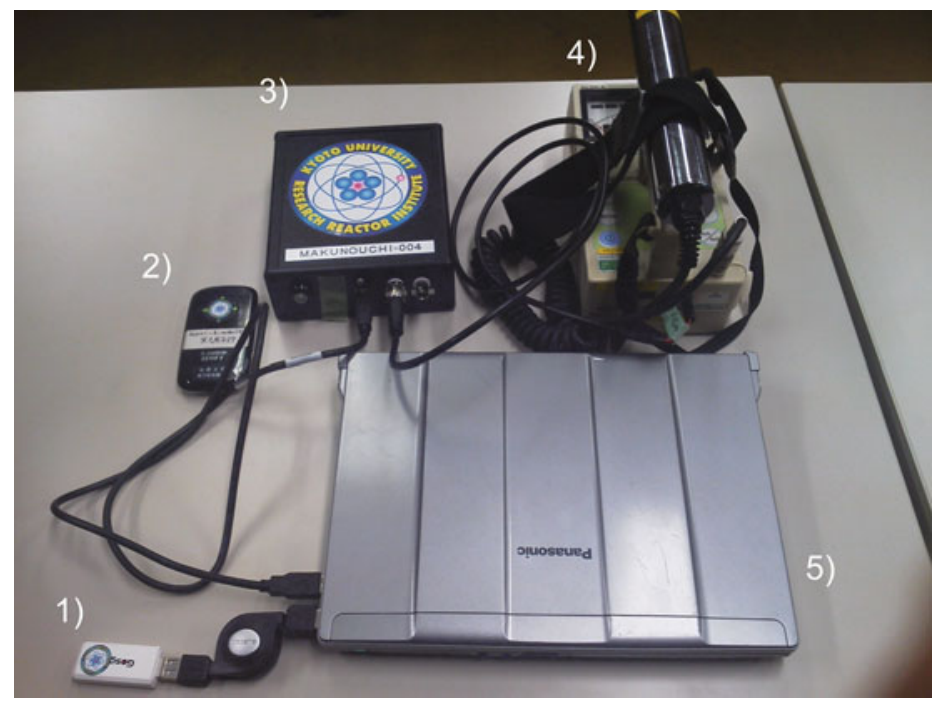

Fig. 10.2 The in-vehicle part is compactly composed of mostly commercial components. (1) GPS unit, (2) 3G mobile Wi-Fi router, (3) MAKUNOUCHI, (4) NaI survey meter, and (5) PC 


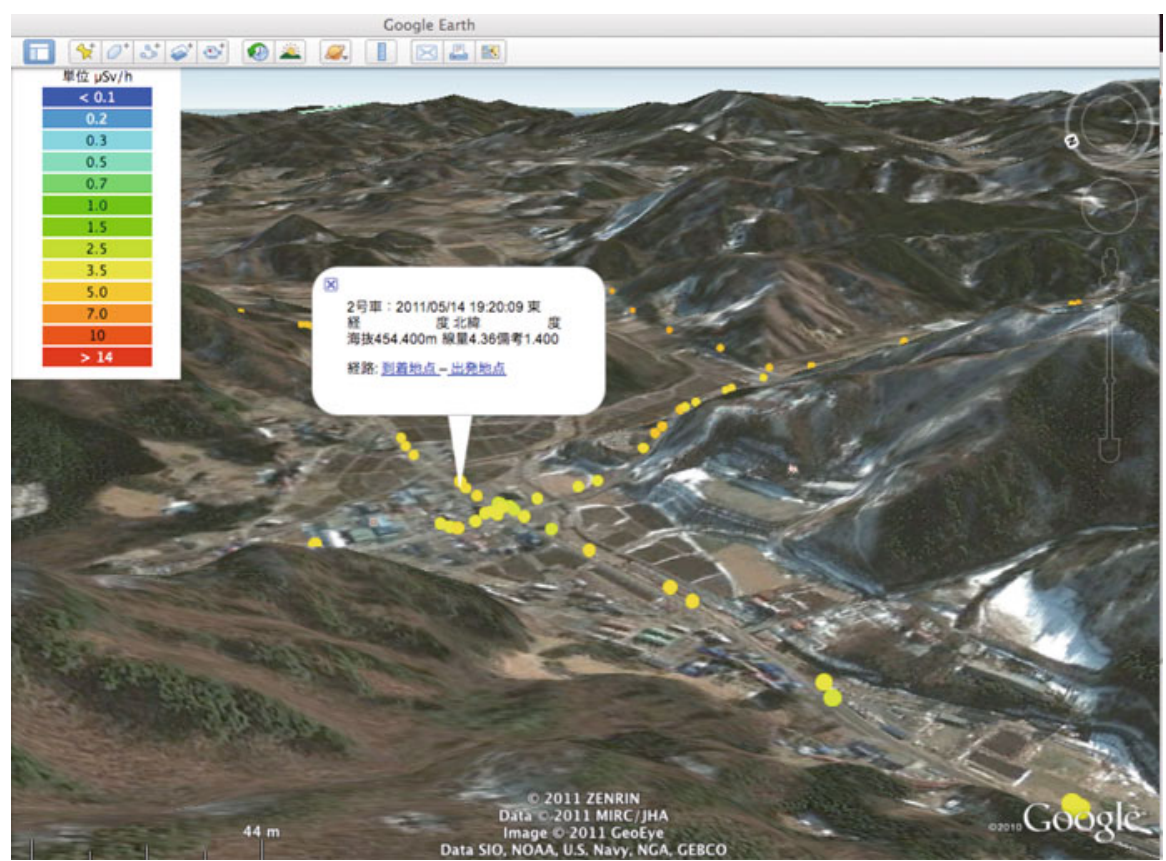

Fig. 10.3 The data is simultaneously plotted on Google Earth. The color of each dot represents the air dose rate at respective point

collection. With this feature, anyone can set up their own "data center" anywhere as far as a conventional Internet connection and a PC with Dropbox are available. This kind of flexibility should be required in disasters like the present case because the carborne survey system owned by Fukushima prefecture eventually came to a halt due to the shutdown of the data center by the disaster.

Once the radiation data in csv format is shared with remote servers, the data file is processed by servers in various ways, including the real-time display on Google Earth in client PCs (Fig. 10.3).

\subsection{KURAMA-II}

Long-term (several tens years) and detailed surveillance of radiations are required in residential areas that are exposed to radioactive materials. Such monitoring can be realized if moving vehicles in residential areas such as buses, delivery vans, or bikes for mail delivery have KURAMA onboard. KURAMA-II [11] is designed for such purpose. 


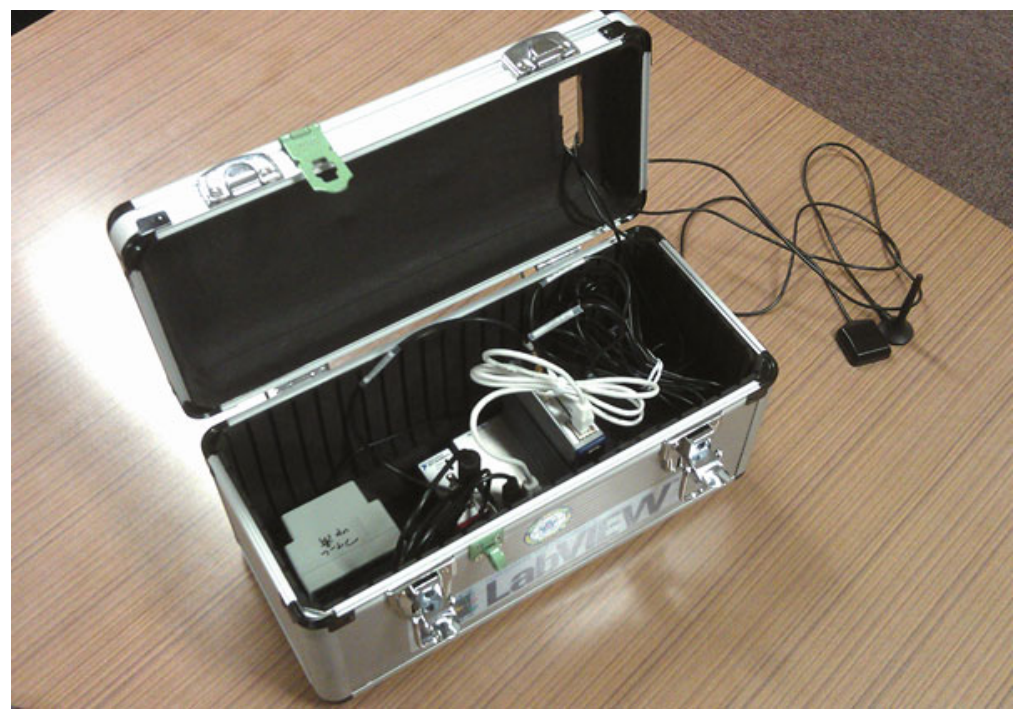

Fig. 10.4 The in-vehicle unit of KURAMA-II. A CsI detector and a CompactRIO are compactly placed in a toolbox with the size of $34.5 \times 17.5 \times 19.5 \mathrm{~cm}$

KURAMA-II stands on the architecture of KURAMA, but the in-vehicle part is totally redesigned. The platform is based on CompactRIO series by National Instruments to obtain better toughness, stability, and compactness. The radiation detection part is replaced from the conventional NaI survey meter to a Hamamatsu C12137 detector [4], a CsI detector characterized by its compactness, high efficiency, direct ADC output, and USB power operation. The direct ADC output enables to obtain $\gamma$-ray energy spectra during operation. The mobile network and GPS functions are handled by a Gxxx 3G series module for CompactRIO by SEA [5]. All of the components for the in-vehicle part are placed in a small toolbox for a better handling (Fig. 10.4).

The software for KURAMA-II is basically the same code as that of original KURAMA, thanks to the good compatibility of LabVIEW over various platforms. Additional developments were performed in several components such as device control software for newly introduced C12137 detector and Gxxx 3G module, the start-up and initialization sequences for autonomous operation, and the file transfer protocol.

CompactRIO is designed for applications in harsh environment and limited space. Therefore, KURAMA-II can be used other than carborne surveys (Fig. 10.5). For example, KURAMA-II is loaded on a motorcycle intending not only the attachment with motorcycles for mail delivery, but also the monitoring in regions where conventional cars cannot be driven, such as small paths between rice fields or those through forests. Also, KURAMA-II with DGPS unit is prepared for the precise mapping by walking in rice fields, orchards, parks, and playgrounds in Fukushima. 

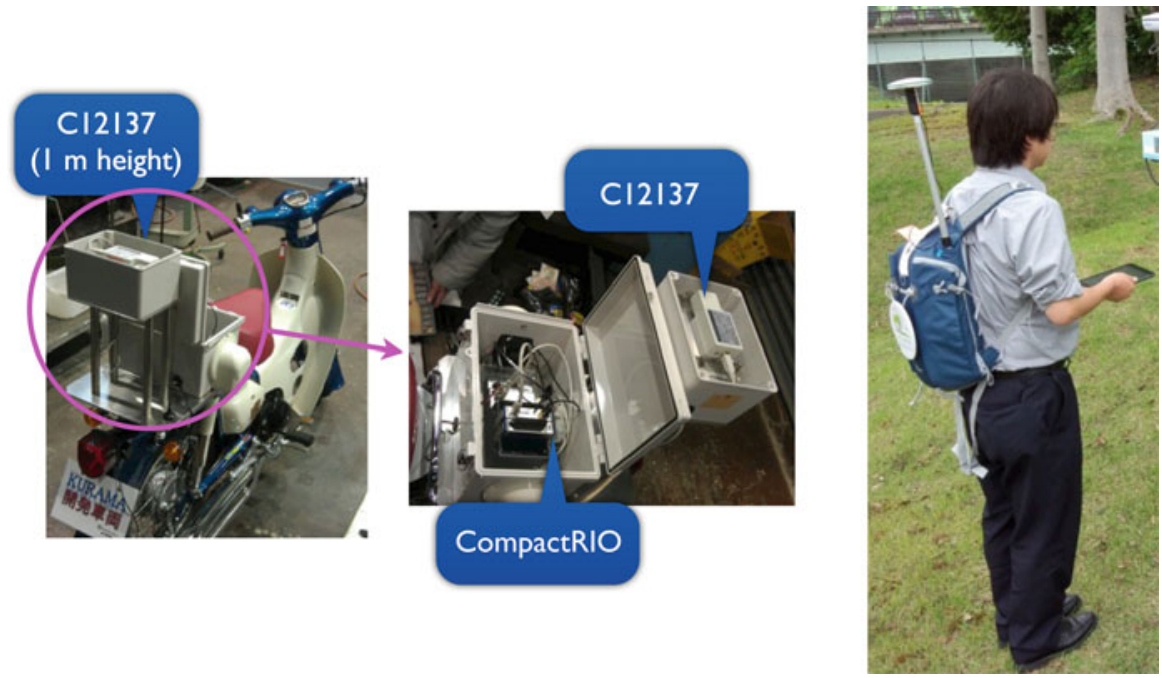

Fig. 10.5 KURAMA-II for bike survey (left and middle) and walking survey (right). All have basically the same hardware and software configuration with different ways of installation. In the case of walking survey, the existing GPS part is replaced with DGPS for the better precision of positioning measurement

\subsection{Applications of KURAMA and KURAMA-II}

As the developer of KURAMA and KURAMA-II, we have demonstrated possible applications of KURAMA and KURAMA-II through a series of field tests. In the beginning, we demonstrated an efficient $\gamma$-ray carborne survey by KURAMA in Fukushima prefecture in collaboration with the Fukushima prefectural government (Fig. 10.6). This result encourages the Ministry of Education, Culture, Sports, Science and Technology in Japan (MEXT) to conduct the first official carborne survey project by KURAMA.

We then carried out a field test of continuous monitoring by KURAMA-II on a local bus in Fukushima city in December 2011 in collaboration with Fukushima Kotsu Co. Ltd., one of the largest bus operators in Fukushima prefecture (Fig. 10.7). Local buses are suitable for continuous monitoring purpose because of their fixed routes in the center of residential areas and routine operations.

Based on the success of the field test on a local bus in Fukushima city, the region of this field test has been extended to other major cities in Fukushima prefecture since January 2013, i.e., Koriyama city, Iwaki city, and Aizuwakamatsu city. Five KURAMA-II in-vehicle units are deployed for this test, and the result is summarized and released to the public from the website [6] on a weekly basis.

The team of the Fukushima prefectural government made precise radiation maps of major cities in Fukushima prefecture mainly for "hot spot" search just after KURAMA was available [3]. Soon, the Fukushima prefectural government sought the possibility to extend the radiation monitoring by local buses over Fukushima 


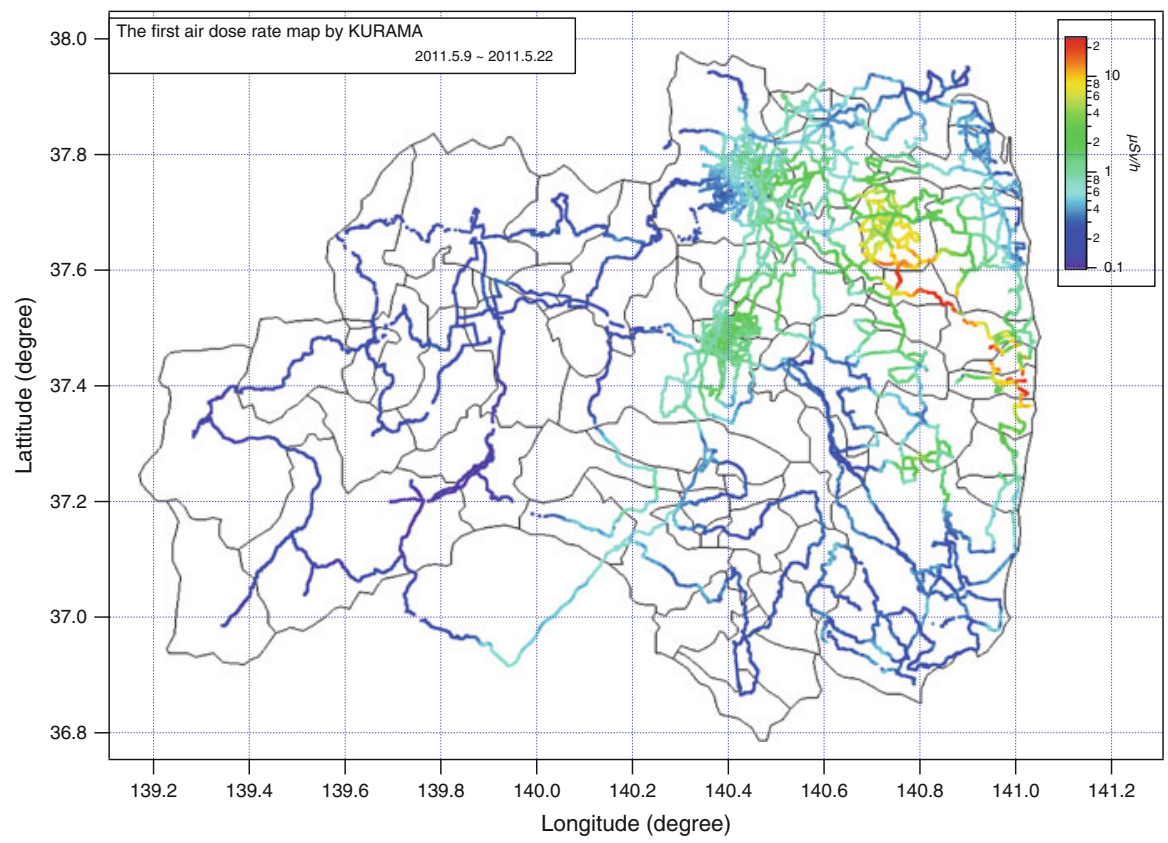

Fig. 10.6 The air dose rate map generated by the first demonstration of KURAMA in May 2011. This was also the first prefecture-wide map of air dose rate in this accident

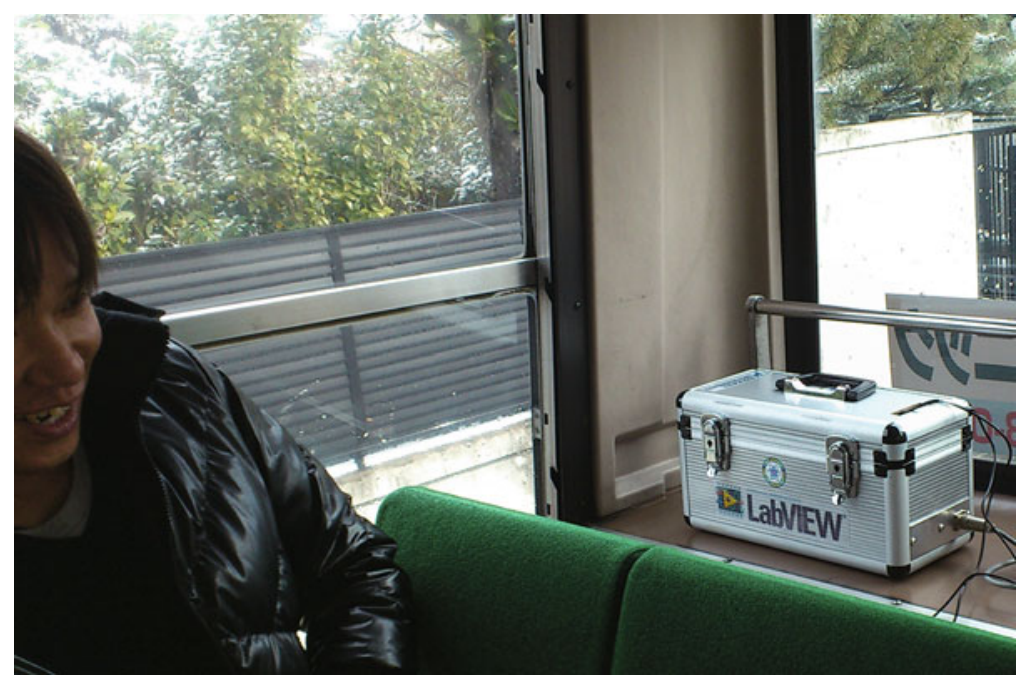

Fig. 10.7 KURAMA-II under the field test on a local bus 


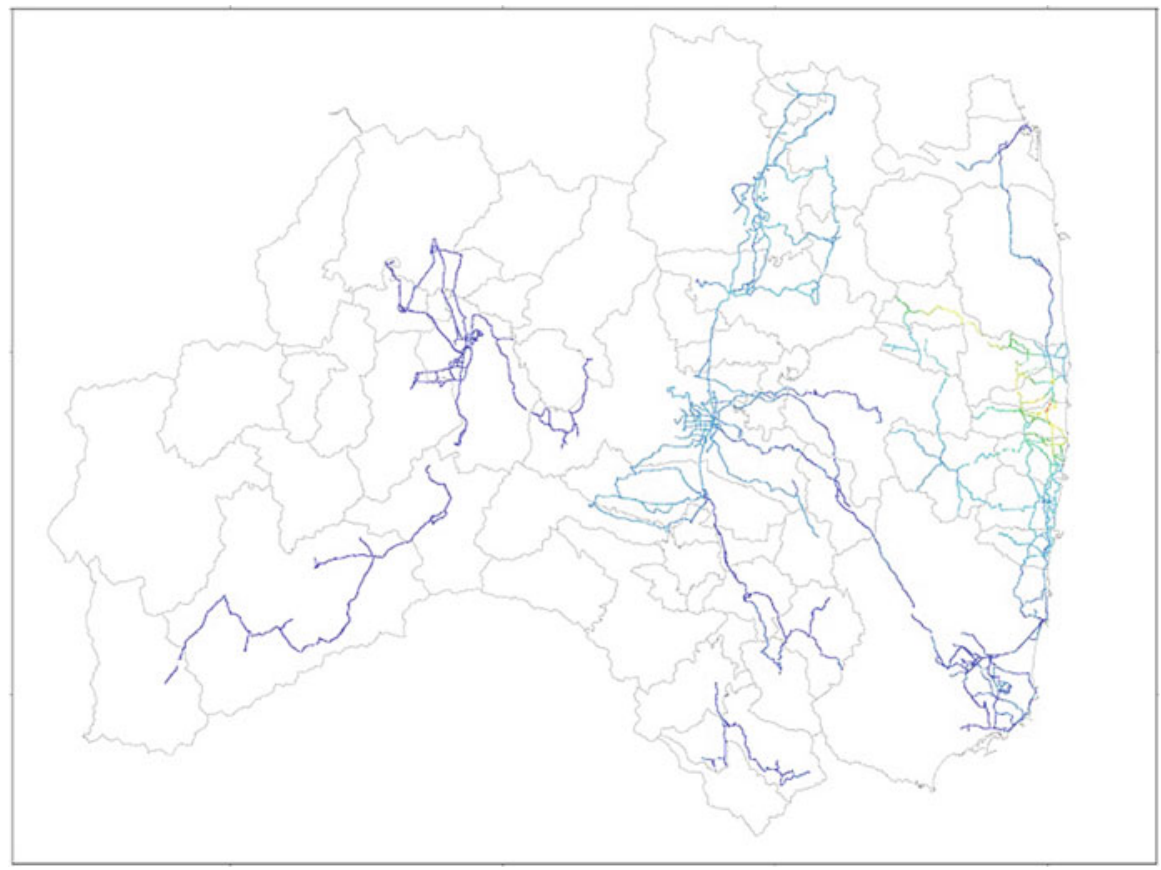

Fig. 10.8 A result of the continuous monitoring by KURAMA-II on local buses conducted by the Fukushima prefectural government [1]

prefecture because they realized the importance of continuous monitoring in residential areas. In August 2015, the monitoring scheme by local buses started as an official operation by the Fukushima prefectural government with the collaboration of Kyoto University and Japan Atomic Energy Agency (JAEA). As of May 2015, 30 local buses owned by bus companies and 20 official cars owned by Fukushima prefecture are continuously operated throughout Fukushima prefecture. Real-time data is released to the public on the display system at the public space of a building in Fukushima city, and the summarized results are available on a weekly basis on the web (Fig. 10.8) [1].

MEXT conducted the very first official carborne survey in Fukushima prefecture and its surrounding area in June 2011. Then they extended carborne surveys in Eastern Japan [8] including Tokyo metropolitan area in December 2011. MEXT started a carborne survey project in March 2012, in which 100 units of KURAMAII were lent to municipalities in Eastern Japan [7]. KURAMA-II was placed in sedan cars of municipalities, and the cars were driven around by ordinary staff members in respective municipalities, who didn't have any special training on radiation measurement. This survey was successful and proved the performance and scalability of KURAMA-II system. Now, this survey has been conducted periodically by MEXT and the national regulation authority (NRA), which is the successor of the radiation monitoring of the present incident (Fig. 10.9). 


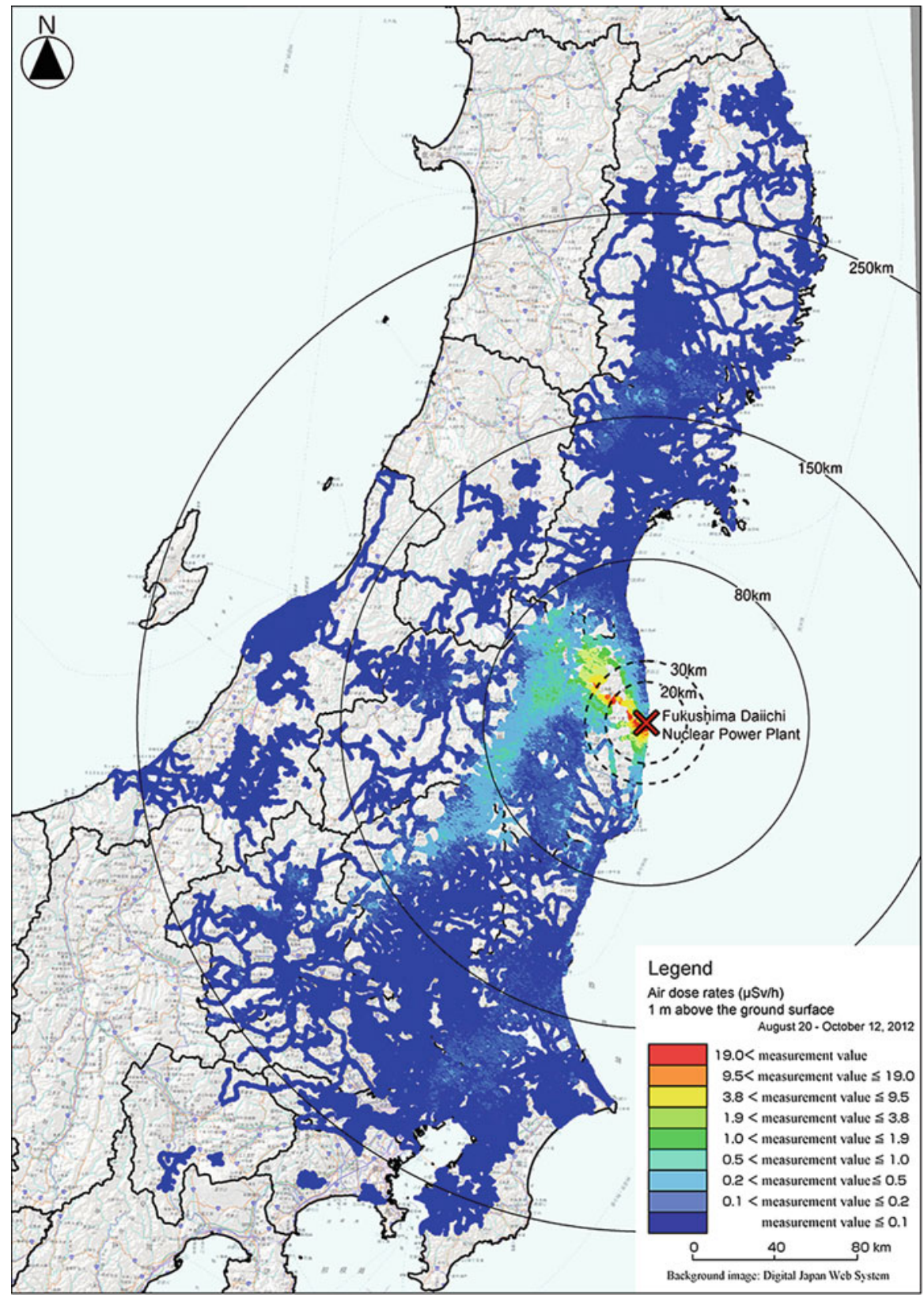

Fig. 10.9 Map of air dose rates on roads measured by KURAMA-II in the periodical survey conducted by NRA between August and October 2012 [9] 
Acknowledgements The author is grateful to Dr. Mizuno, Mr. Abe, Mr. Koyama, and the staff members of the KURAMA operation team at the Fukushima prefectural government for their continuous support to the field tests of KURAMA in Fukushima. The author is thankful to Dr. Ito of ICR, Kyoto University; Prof. Maeno of Graduate School of Science, Kyoto University; and the staff members of the KURAMA field test team of RRI, Kyoto University for their contribution to the test operation in Fukushima. The author is indebted to Dr. Saito, Mr. Yoshida, and Dr. Takemiya at JAEA for the discussions concerning the operation of KURAMA and to Dr. Tsuda at JAEA for evaluating the $G(\mathrm{E})$ functions of $\mathrm{C} 12137$ series. The development of KURAMA-II is adopted by "Japan recovery grant program" from National Instruments, Japan. The field tests of KURAMA-II on local buses are supported by Fukushima Kotsu Co. Ltd., Shin Joban Kotsu Co. Ltd., and Aizu Noriai Jidosha Co. Ltd. The author is indebted to Mr. and Mrs. Takahashi and the staff members at Matsushimaya Inn, Fukushima, for their heartwarming hospitality during the activities in Fukushima regardless of their severe circumstances due to the earthquake and the following nuclear accident.

Open Access This chapter is distributed under the terms of the Creative Commons Attribution Noncommercial License, which permits any noncommercial use, distribution, and reproduction in any medium, provided the original author(s) and source are credited.

\section{References}

1. Fukushima Prefectural Government (2014) Vehicle-borne survey monitoring in fukushima prefecture, Jan 2014. https://www.pref.fukushima.lg.jp/sec/16025d/soukou.html (in Japanese)

2. Guidelines for Radioelement Mapping Using Gamma Ray Spectrometry Data, IAEATECDOC-1363, International Atomic Energy Agency, 2003, p 40

3. Fukushima Prefectural government (2011) Air dose measurement by KURAMA. http://www. pref.fukushima.lg.jp/sec/16025c/genan28.html (in Japanese)

4. Hamamatsu Photonics Inc (2011) Radiation detection module. http://www.hamamatsu.com/jp/ en/product/category/3100/4012/4134/C12137/index.html

5. S.E.A. gmbh (2011) SEA 9724 product flyer. http://www.sea-gmbh.com/en/products/ compactrio-products/mobile-communications/sea-9724-3ggps/

6. Research Reactor Institute, Kyoto University (2012) Field test of KURAMA-II. http://www. rri.kyoto-u.ac.jp/kurama/kouiki/kurama2_test.html (in Japanese)

7. Ministry of Education, Culture, Sports, Science and Technology (2012) Results of continuous measurement of air dose rates through a vehicle-borne survey (as of March 2012). http://radioactivity.nsr.go.jp/en/contents/6000/5637/24/338_Suv_091218_e.pdf

8. Press Release (2012) Results of continuous measurement of air dose rates through a vehicleborne survey by MEXT (as of Dec 2011), 21 Mar 2012, MEXT. http://radioactivity.mext.go. jp/en/contents/5000/4688/view.html

9. Saito K (2013) Studies on the precise distribution and trends of air dose rate on the road by using carborne survey technique - the introduction of the progress of the third distribution study, Feb 2013. http://www.jaea.go.jp/fukushima/kankyoanzen/tyouki-eikyou/giji/08/pdf/82-1.pdf (in Japanese)

10. Tanigaki M, Okumura R, Takamiya K, Sato N, Yoshino H, Yamana H (2013) Development of a carborne $\gamma$-ray survey system, KURAMA. Nucl Instrum Methods Phys Res Sect A 726: $162-168$

11. Tanigaki M, Okumura R, Takamiya K, Sato N, Yoshino H, Yoshinaga H, Kobayashi Y, Uehara U, Yamana H (2015) Development of KURAMA-II and its operation in Fukushima. Nucl Instrum Methods Phys Res Sect A 781:57-64 